\title{
La lutte pour la terre macédonienne : un projet grec de 1909
}

The fight for Macedonian land: a greek plan of 1909

Spyros Karavas

\section{(2) OpenEdition}

\section{Journals}

Édition électronique

URL : https://journals.openedition.org/ceb/1041

DOI : 10.4000/ceb.1041

ISSN : 2261-4184

Éditeur

INALCO

\section{Édition imprimée}

Date de publication : 9 janvier 2012

ISSN : 0290-7402

\section{Référence électronique}

Spyros Karavas, «La lutte pour la terre macédonienne : un projet grec de 1909 », Cahiers balkaniques [En ligne], 40 | 2012, mis en ligne le 25 mai 2012, consulté le 06 juillet 2021. URL : http:// journals.openedition.org/ceb/1041; DOI : https://doi.org/10.4000/ceb.1041

Ce document a été généré automatiquement le 6 juillet 2021

\section{(c) (†) 8}

Cahiers balkaniques est mis à disposition selon les termes de la Licence Creative Commons Attribution - Pas d'Utilisation Commerciale 4.0 International. 


\title{
La lutte pour la terre
} macédonienne : un projet grec de 1909

The fight for Macedonian land: a greek plan of 1909

\author{
Spyros Karavas
}

\section{NOTE DE L'ÉDITEUR}

Voir S. Karavas, « Bienheureux ceux qui possèdent la terre », projets fonciers en vue de l'expropriation des consciences en Macédoine, 1880-1909 (en grec), Athènes, Vivliorama, 2010.

1 J'ai choisi de commencer par une petite anecdote historique. En mars 1908, quelques mois avant l'avènement des Jeunes-Turcs, un employé de l'ambassade grecque à Rome publia dans un journal italien un article sur la politique du royaume de Grèce en Macédoine. Bien que Grec, l'auteur accusait son gouvernement de dépenser des millions pour une armée incapable, apathique et démoralisée. On croyait dans le royaume que cette armée pourrait un jour conquérir la Macédoine et la rattacher à la Grèce. Or, soulignait l'auteur de l'article, c'était impossible parce que les autochtones non seulement ne coopéraient pas avec les bandes envoyées de Grèce, mais, au contraire, soutenaient les bandes bulgares ennemies.

2 Il ressort de la correspondance consulaire relative à l'affaire Ladikos (du nom de l'auteur de l'article) que cet employé, pour des raisons qui n'ont pas été éclaircies, était en conflit avec l'ambassade où il servait depuis 1904, peut-être à titre non officiel, et qu'à présent il se vengeait en écrivant des articles dans la presse italienne. En l'occurrence, les accusations de Sp. Ladikos seraient restées parfaitement anodines si elles n'avaient été suivies d'une violente réaction de l'ambassade grecque. Réaction qui culmine avec la procédure d'expulsion d'Italie de Ladikos comme criminel de droit commun ayant abusé du titre d'agent politique. Telle est la version officielle, la plainte 
de l'ambassade formulée auprès des autorités italiennes pour obtenir l'expulsion. Mais ce qui nous intéresse ici, c'est la raison véritable de la sanction, telle que l'ambassadeur l'avoue: Ladikos «a remué sur une terre libre (c'est-à-dire l'Italie) les plaies de sa patrie ». C'est ce qu'il écrit à son ministre en juin $1908^{1}$.

3 Car la politique de l'État grec sur la question macédonienne était effectivement une plaie béante que le changement politique causé par les Jeunes-Turcs, comme certains l'espérèrent à tort, ne put aider à se refermer. Un changement qui, ne l'oublions pas, fut précipité en juillet 1908 pour garder la Macédoine entre des mains turques, suite à la rencontre anglo-russe à Reval (Tallinn) qui, en juin de la même année, avait remis en cause la souveraineté turque sur elle ${ }^{2}$.

4 Mais si la Turquie trouva le moyen, avec l'arrivée des Jeunes-Turcs, de sauver la Macédoine pour son compte, fût-ce de manière provisoire, les inquiétudes grecques ne cessèrent de croître au fur et à mesure que s'accumulaient les messages décourageants. Alors que, tant qu'avait duré l'état de guerre informel, jusqu'en juillet 1908, beaucoup de villages bulgarophones, sous la menace des armes, étaient restés sous surveillance grecque, maintenant ils se rangeaient massivement du côté de l'adversaire. Comme l'expliquent les chargés d'affaires grecs à leurs supérieurs (le ministère des Affaires étrangères), cette évolution n'était pas du tout fortuite. Depuis août 1908, aussi bien l'Organisation révolutionnaire macédonienne intérieure (VMRO) que la fraction de Sandanski en Macédoine Orientale s'activaient en faveur de la population rurale locale, demandant à la nouvelle direction politique de concrétiser ses revendications constantes, parmi lesquelles figuraient le remembrement de la terre et l'attribution de celle-ci à ceux qui la cultivaient ${ }^{3}$.

\section{Le plan de Saktouris}

5 Au début de 1909, le nouveau Parti fédéral national de Sandanski et de Çernopeev, qui conservait une grande influence sur la population de Strumnitsa et de Serrès, prêche la réforme agricole tout en maintenant, du moins en surface, d'excellentes relations avec le comité Union et Progrès des Jeunes-Turcs ${ }^{4}$. Naturellement, l'écho que rencontrent les déclarations de Sandanski et des partisans des Bulgares inquiète au plus haut point le consul grec de Serrès, Antonios Saktouris. En février 1909, il estime que la victoire finale, pour ce qui est de la Macédoine, reviendra aux "Bulgares», parce que "la plupart des choses jouent en leur faveur et très peu en la nôtre $»^{5}$.

Le pessimisme de Saktouris est dû au fait que, en dehors du soutien qu'ils ont réussi à obtenir des Jeunes-Turcs, les autonomistes du VMRO font la tournée des villages et des tsifliks et, faisant miroiter l'appât essentiel qu'est la promesse du partage des terres, parviennent à convaincre les cultivateurs naïfs d'intégrer les rangs des autonomistes des "Bulgares", selon Saktouris. Dans le même temps, les constatations du consul concernant la supériorité numérique des paysans bulgarophones par rapport aux minorités grecques, y compris dans la plaine située au sud de Serrès, d'une part, et d'autre part le zèle et la frugalité des bulgarophones, en concurrence avec les hellénophones, aggravent ses inquiétudes. C'est précisément pour cette raison qu'il confesse au ministre grec des Affaires étrangères qu'il envisage avec effroi l'avenir des intérêts grecs en Macédoine.

Quelque temps plus tard (au début mars 1909), Saktouris reviendra sur l'enjeu majeur, à savoir la question rurale en Macédoine, qui est selon lui la clé de tout le problème. Pour 
lui, le plan «diabolique » des Bulgares, mis en œuvre depuis la dernière décennie au moins, consistait à «donner à la question rurale de la Macédoine un caractère national». En d'autres termes, ils persuadaient les serfs bulgarophones, qui constituaient de toute façon, comme le consul de Serrès est le premier à l'admettre, la majorité de la population chrétienne de la Macédoine, que les terres qu'ils cultivaient leur appartenaient historiquement et seraient à eux s'ils en chassaient les propriétaires actuels, les beys turcs et les Grecs. Ainsi, poursuit Saktouris, les Bulgares avaient-ils associé l'idée de la liberté à celle de l'indépendance matérielle « de ces êtres la plupart de temps réellement malheureux $»^{6}$.

8 Le résultat de la propagande bulgare - de toutes tendances, autonomiste ou non -, qui parvint à associer la libération nationale à l'émancipation sociale, fut la victoire manifeste du bulgarisme et la perspective d'une défaite définitive de l'hellénisme. Aujourd'hui, souligne le consul, toute la zone revendiquée se transforme en région bulgare, alors que c'était une région fidèle au régime ottoman. Les Bulgares, et en particulier Sandanski, en contact permanent avec le comité Jeune-Turc, obligeront tôt ou tard celui-ci à procéder au remembrement de la terre. Et alors, " pour nous ", note Saktouris, tout sera fini.

9 Sans le savoir, le consul grec de Serrès donnait tout à fait raison à René Pinon qui, en été 1908, écrivait à propos de la question macédonienne :

"La question macédonienne est à la fois une question sociale et une question nationale : pour la résoudre, il faudrait toucher au régime de la propriété et au régime de la souveraineté, c'est-à-dire au tuf même de l'organisation turque en Macédoine »?

10 Mais là où Saktouris se démarque de René Pinon, c'est sur le remède à appliquer. Pour Saktouris, il n'y avait qu'une seule solution, un seul remède pour contrer le désastre imminent qui menaçait l'hellénisme. Il consistait à acheter des tchifliks avec des fonds prélevés sur le budget de l'État grec, et à les partager entre les serfs. Ces derniers acquerraient la propriété de leur terre au bout de dix à vingt ans, en versant chaque année la somme correspondante à l'acheteur virtuel, qui devait être impérativement un Grec ressortissant ottoman.

11 Pendant ces vingt ans, ajoute Saktouris, non seulement les populations bénéficiaires n'oseraient pas faire défection et se détacher de l'hellénisme, mais parallèlement, le mécanisme de propagande que la Grèce conservait en Macédoine (avec l'Église et les écoles) aurait l'occasion de préparer "une nouvelle génération purement grecque ». Pendant la même période, et jusqu'à ce que ces Grecs purs soient "fabriqués ", Saktouris estime nécessaire d'établir, à côté des Macédoniens bulgarophones, des paysans hellénophones du Caucase, des Pontiques de la mer Noire ou d'autres immigrants. Ainsi la population purement bulgare de la plaine de Serrès deviendraitelle mixte. La qualification de "mixte» ne vaudrait manifestement que durant cette période décisive de vingt ans, car ensuite, grâce à la création de la nouvelle génération purement grecque, comme dit plus haut, l'ensemble de la population serait désormais de conscience purement grecque.

12 Terminant son rapport, Saktouris note qu'il est impossible d'acheter tous les tchifliks du district de son consulat, surtout d'ailleurs que les prix avaient atteint des sommets depuis l'avènement des Jeunes-Turcs, et qu'il fallait donc sélectionner les domaines d'importance stratégique. 


\section{Une idée répandue...}

13 Cette proposition machiavélique, impliquant le double processus de privatisation des terres et d'immigration, n'a bien sûr pas été inventée par les Grecs, elle n'est pas nouvelle dans les milieux nationalistes grecs et ne concerne pas seulement le sandjak de Macédoine orientale. Expliquons-nous.

Commençons par ce dernier aspect : à la même époque, en novembre 1908, un autre agent du gouvernement grec à Thessalonique propose à la nation de se hâter d'acheter des tchifliks dans la région comprise entre Vodena et Gianitsa afin de maintenir les paysans métayers hors de la portée des organisations bulgares. Parallèlement, il estime nécessaire, tout comme Saktouris, de veiller à installer des paysans hellénophones venus de l'ancienne Roumélie orientale ${ }^{8}$. On trouve expressément des recommandations analogues dans le courrier officiel, souvent formulées non sans cynisme. Mais on rencontrera aussi des incitations similaires dans des publications ordinaires. En tout état de cause, ici, le discours est dissimulé et le message est diffusé sous le couvert d'une rhétorique qui prône le développement économique.

Je disais précédemment que le projet concret d'hellénisation de la Macédoine n'était pas nouveau dans les milieux grecs. En effet, il fait partie de l'arsenal du nationalisme grec relatif à la politique exercée en Macédoine depuis au moins trente-cinq ans. En particulier, dès 1880, c'est un leitmotiv dans le discours des dirigeants grecs et le stratagème suprême pour infiltrer et conquérir pacifiquement l'espace macédonien revendiqué'. Pendant près de trente-cinq ans, l'objectif restera le même : l'hellénisation de la Macédoine par l'appropriation des terres, par l'assujettissement, en parallèle, de ses cultivateurs bulgarophones et par l'immigration de paysans, autant que possible hellénophones. Cependant, les mécènes de la diaspora grecque ne prirent pas à un degré suffisant le risque «national » de placer leurs capitaux dans les sables mouvants de la terre par ailleurs fertile de la Macédoine, qui d'ailleurs, selon toutes les estimations, était sous-évaluée.

16 Enfin, comme je l'ai dit, ce double processus de nationalisation des terres par le biais du rachat et de l'installation d'une population rurale aux dispositions amicales n'est pas grec et n'a pas été inventé en Orient. Cette idée se développa pendant les années 1870 dans l'Allemagne tout juste unifiée, pour être appliquée par Bismarck au cours de la décennie suivante et se transmettre, entre autres, aux milieux nationalistes grecs de l'époque. En d'autres termes, c'est principalement le fruit du nationalisme allemand, qui trouva à s'appliquer dans les territoires polonais de Prusse-Orientale, principalement dans les années 1885 à $1887^{10}$.

17 Notons que, bien que l'entreprise de germanisation de la Pologne occupée n'ait pas cessé jusqu'en 1918, le processus échoua (voir R. L. Koehl, 1953) parce que, entre autres, les grands propriétaires fonciers allemands préféreraient la main-d'œuvre polonaise, peu coûteuse, pour cultiver la terre. Comme le soulignait alors de manière significative le jeune Max Weber, accusant précisément les grands propriétaires fonciers allemands, "le plus grand polonisateur» de la Prusse-Orientale, ce fut les grandes propriétés foncières ${ }^{11}$. Rappelons ici que l'un des reproches faits par les responsables grecs aux propriétaires fonciers grecs, de toute façon peu nombreux en Macédoine, c'était de préférer employer la main-d'œuvre bon marché issue de la population bulgarophone. 
18 L'échec de l'hellénisation de la Macédoine pendant cinquante ans de suite, que ce soit par la voie de l'appareil ecclésiastique et éducatif ou par l'hellénisation de la terre, par l'infiltration économique et par l'immigration, furent sans aucun doute pour les dirigeants grecs la plus grande " plaie », pour reprendre l'expression de l'ambassadeur de Grèce à Rome.

19 Cette plaie, c'est-à-dire la supériorité slave en Macédoine, ne laissa certes pas indifférentes les puissances européennes qui vivaient dans une ambiance de panslavophobie, la première d'entre elles étant l'Angleterre. Bien avant que les Allemands n'accomplissent leur unité nationale et ne mettent au point leurs idées pangermaniques, les diplomates anglais seront peut-être les premiers à s'inquiéter de l'altération de la « chimie nationale » de la Macédoine. En effet, dès 1869, ils tenteront de persuader la Sublime Porte d'autoriser l'installation, sur les terres non cultivées de Macédoine, des paysans irlandais ruinés qui avaient envahi les villes anglaises ou partaient pour l'Amérique ${ }^{12}$. Le refus de la Porte privera la Macédoine d'une colonisation irlandaise, et la trouvaille européenne de la salade dite « macédoine » aura une composition exclusivement orientale.

\section{Un plan irréalisable}

20 Mais pour en revenir à la Macédoine des Jeunes-Turcs, s'il y a maintenant une nouveauté, après le changement politique du régime turc, c'est le contenu en lui-même des projets en ce domaine et, en outre, le caractère d'urgence qu'ils acquièrent. C'est ainsi que, alors qu'au cours des années précédentes, les principaux destinataires de la demande de rachat étaient les Grecs riches de l'étranger, maintenant, en 1908-1909, sous la pression de la conjoncture, le seul destinataire est le royaume grec et son budget.

21 Une seconde différence réside dans la rentabilité économique de l'entreprise. Cette rentabilité, qui avait été vantée à l'extrême, cesse d'être une réalité en 1908. Aussi bien l'augmentation brutale du prix de la terre que l'urgence nationale ne laissaient plus de marge aux bonnes affaires. Le processus de développement avec des capitaux grecs qui avait été programmé depuis des années pour modifier le sentiment des populations rurales n'avait pas réussi. Maintenant, au dernier moment, l'État grec était appelé à acheter des terres sur un territoire toujours étranger et à les partager à ses cultivateurs avec un amortissement à long terme. Un remembrement inopiné, semblable à un tour de passe-passe, qu'il fallait réaliser en catimini, avec des acheteurs fictifs.

Qui, en effet, aurait convaincu les serfs que leur bienfaiteur était le royaume grec ? Et cela au moment même où ce dernier s'abstenait de toute initiative politique sur la question du côté du comité Union et Progrès, analogue à celle de Sandanski et du VMRO. Au moment même où le royaume grec, de par sa position, ne pouvait nullement s'ériger en porte-parole du mouvement social et rural en Macédoine. D'ailleurs, le slogan : «La terre macédonienne aux Macédoniens » était depuis des années scandé par l'ennemi. Comment aurait-il été possible qu'il se traduise en actes de la part de ceux qui l'avaient combattu, ceux qui, en coopération avec les Ottomans, avaient lutté de toutes leurs forces contre toute idée d'autonomie de la Macédoine? Saktouris connaissait parfaitement ces questions sans réponses. Mais devant l'impasse, il tenta de sauver ce qui pouvait l'être, c'est-à-dire de sauvegarder in extremis ne serait-ce qu'un minimum des revendications grecques en Macédoine. Il le disait très justement : si elle n'était pas 
résolue par la guerre ou par un "accord radical entre les Puissances européennes », la question macédonienne serait réglée par la résolution de la " question agraire »"

Le plan de Saktouris, indicatif des manœuvres grecques au cours de la période qui nous occupe aujourd'hui, ne fut pas réalisé. Peut-être parce qu'il était trop coûteux, peutêtre faute de temps, ou parce qu'il était de toute façon irréalisable. Ce qui fut réalisé, de manière fragmentaire et à petite échelle, et pour cette raison sans apporter les résultats escomptés, c'est le plan de turquisation de la Macédoine par les Jeunes-Turcs. Quand l'aile nationaliste s'imposa au sein du comité Union et Progrès et que Talaat bey, Sevket pacha et Nazim bey prirent le pouvoir en 1910, ils consacrèrent des fonds de l'ordre de 200000 lires à l'achat de terres et installèrent des musulmans bosniaques en Macédoine, contraignant certains paysans des lieux à partir pour la Bulgarie. Il n'empêche que l'entreprise globale d'« ottomanisation » suivie par Talaat échoua et que c'est d'abord cet échec qui lui coûta le pouvoir en février $1911^{14}$.

En fin de compte, c'est le recours aux armes qui vint ensuite bouleverser de fond en comble la situation, avec les échanges de population qui s'ensuivirent et l'établissement massif de réfugiés en Macédoine grecque.

\section{BIBLIOGRAPHIE}

Aulneau, Jean, (1913), « La question macédonienne », dans Les aspirations autonomistes en Europe. Leçons faites à l'École des Hautes Études Sociales, Paris, pp. 223-224 (texte rédigé en avril 1912).

Chichkov, Stoio N, (1918), Les Bulgares de la plaine de Serrès, (en cyrillique), Plovdiv. On trouvera une traduction française de ce rapport dans 1980, La Macédoine. Recueil de documents et matériaux, Sofia : Académie Bulgare des Sciences, pp. 593-596.

Dakin, Douglas, (1996), La lutte grecque en Macédoine 1897-1913 (en grec), trad. grecque, Thessalonique, (1966 en anglais).

Georgeon, François, (1989), « Le dernier sursaut (1878-1908) », dans R. Mantran (dir.), Histoire de l'Empire ottoman, Paris : Fayard.

Girault, René, (1996), Matériaux pour l'histoire de notre temps, $n^{\circ} 41-42$, Nations, nationalités et nationalismes en Europe de 1850 à 1920, Paris.

Ivanoff, Iordan, (1920), La question macédonienne, Paris : Pochon.

Karavas, Spyros, (2010), "Bienheureux ceux qui possèdent la terre », projets fonciers en vue de l'expropriation des consciences en Macédoine, 1880-1909 (en grec), Athènes : Vivliorama.

Koehl, R. L., (1953), “Colonialism inside Germany: 1886-1918”, The Journal of Modern History, t. 25/3, pp. 255-272.

Mazower, Mark, (1993), Inside Hitler's Greece, Princeton: Mazower (ed).

Pinon, René, (1911), L'Europe et la jeune Turquie. Les aspects nouveaux de la Question d'Orient, Paris : Perrin et \&.

Pinon, René, (1908), L’Europe et l'Empire ottoman, Paris : Perrin. 
Reddaway, W. F., J. H. Penson, O. Halecki, et al., (1950), The Cambridge History of Poland. From Augustus II to Pilsudski (1697-1935), Cambridge: Cambridge University Press.

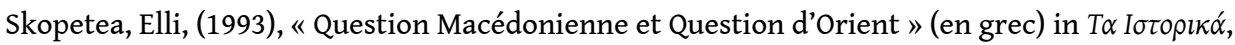
t. 18-19, Athènes.

Skopetea, Elli, (1992), Le déclin de l'Orient. Images de la fin de l'Empire ottoman (en grec), Athènes.

Smotkine, Henri, « La colonisation allemande en Pologne prussienne de 1885 à 1887 », Annales ESC, t. 23/5, p. 1168-1170.

Wonser, Richard, (1941), Germanizing Prussian Poland, The H-K-T Society and the Struggle for the Eastern Marches in the German Empire 1884-1919, New York.

\section{NOTES}

1. Archives du ministère des Affaires étrangères (AMAE), 1908/94.KA', Légation de Grèce en Italie, voir, traduit en grec, l'article publié dans le journal italien par Sp. Ladikos et les lettres de l'ambassadeur de Grèce Chr. Mitsopoulos adressées depuis Rome au ministère des Affaires étrangères en date du 25 mars 1908 (réf. 305) et 8/21 juin 1908 (réf. 443).

2. Concrètement, le 10 juin 1908, lors de la rencontre anglo-russe au sommet à Reval, la GrandeBretagne consentira finalement à l'autonomie de la Macédoine, avec un gouverneur chrétien indépendant du sultan. La réalisation du changement de régime en Macédoine, instamment réclamé par la Bulgarie, la Russie, mais aussi une part importante de l'opinion publique en Europe durant plus de trente ans, devait en fait signifier le début de la fin pour la Turquie. En échange du revirement de la Grande-Bretagne sur la question macédonienne, la Russie renonçait à toute prétention dans le golfe Persique. Ou inversement, comme le note N. Vlachos, « La Russie renonça à toute prétention dans le golfe Persique en achetant le consentement de la Grande-

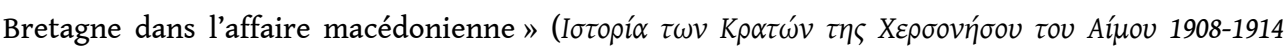
[Histoire des États de la péninsule de l'Aimos], t. 1, Athènes 1954, pp. 30-31, 53). Il va de soi que cette tournure prise par les événements était, aussi bien pour le Patriarcat que pour Athènes, la pire chose qui pouvait se produire. Cependant, le mouvement des Jeunes-Turcs stoppa l'évolution en cours concernant l'affaire macédonienne. Sur le lien direct entre l'accord anglo-russe à Reval et le mouvement jeune-turc, voir J. Aulneau, "La question macédonienne », dans Les aspirations autonomistes en Europe. Leçons faites à l'École des Hautes Études Sociales, Paris 1913, pp. 223-224 (texte

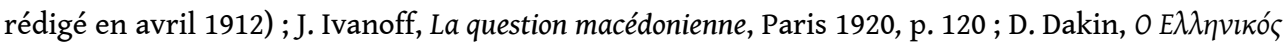

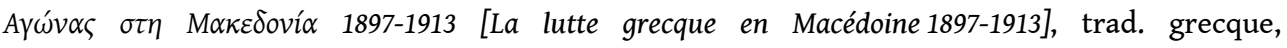
Thessalonique 1996 (11 966), pp. 427, 475, 501 ; Fr. Georgeon, « Le dernier sursaut (1878-1908)»,

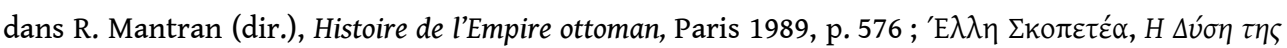

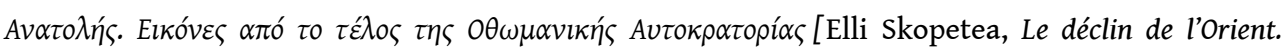

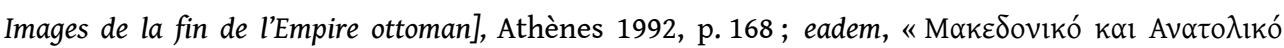

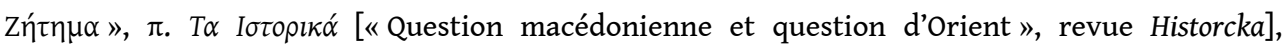
t. 18-19, 1993, p. 149.

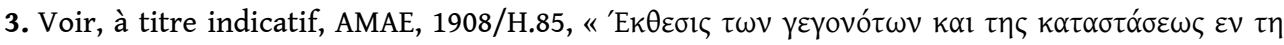

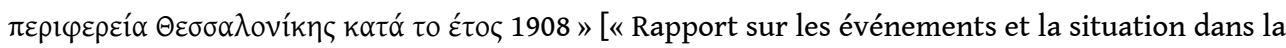
région de Thessalonique durant l'année 1908»] (rédigé par le Consulat général de

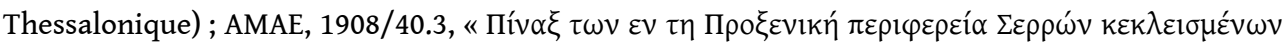

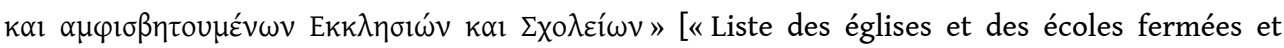

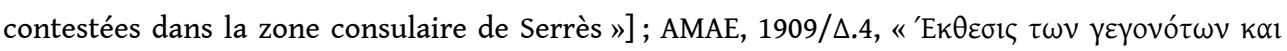

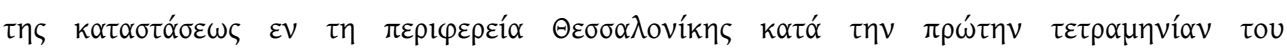


1909 » [ Rapport sur les événements et la situation dans la région de Thessalonique durant les quatre premiers mois de $1909 »]$, daté de mai 1909.

4. D. Dakin, op. cit., pp. 512-522.

5. Archives littéraires et historiques grecques (ELIA) de Thessalonique, Archives d'Angelos Anninos, dossier 1.2, rapport dactylographié daté de Serrès, 28 septembre 1909, réf. 135. Il a été publié, avec en regard une traduction en bulgare, dans l'ouvrage de Yordan Pop-Guéorguiev -

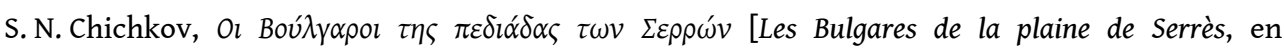
cyrillique], Plovdiv 1918, pp. 40-44. On trouvera une traduction française de ce rapport dans $L a$ Macédoine. Recueil de documents et matériaux, Sofia, Académie bulgare des Sciences, 1980, pp. 593-596.

6. Ce rapport, daté de Serrès, 6 mars 1909, réf. 144, a été publié avec en regard une traduction en bulgare, dans l'ouvrage de Yordan Pop-Guéorguiev - S. N. Chichkov, op. cit., p. 44-48. Traduction française de ce rapport dans La Macédoine. Recueil de documents, op. cit., pp. 597-599.

7. R. Pinon, L'Europe et l'Empire ottoman, Paris 1908, p. 231. Ce même auteur se montre encore plus éloquent en une autre occasion: «La question macédonienne avant d'être une question de nationalité est une question sociale. Je veux dire que c'est la misère des paysans réduits, par les beys Turcs, à la condition de serfs corvéables et taillables à merci, qui fait la situation intolérable de la population chrétienne de Macédoine »: idem, «Les Macédoniens » (1907), reproduit par René Girault, Matériaux pour l'histoire de notre temps, $\mathrm{n}^{\circ} 41-42$, Nations, nationalités et nationalismes en Europe de 1850 à 1920, Paris 1996, p. 32-33. Voir, du même, L'Europe et la jeune Turquie. Les aspects nouveaux de la Question d'Orient, Paris, 1911, p. 129.

8. Bibliothèque Gennadios, Archives Stefanos Dragoumis, dossier 209.1.5 (123), rapport daté de Thessalonique, 21 novembre 1908.

9. Sur ce point, voir en détail Sp. Karavas, op.cit à la note 1, Athènes, 2010.

10. Voir, à titre indicatif, R. Wonser, Germanizing Prussian Poland, The H-K-T Society and the Struggle for the Eastern Marches in the German Empire 1884-1919, New York 1941; W. F. Reddaway, J.H. Penson, O. Halecki, R. Dyboski, The Cambrige History of Poland. From Augustus II to Pilsudski (1697-1935), Cambridge 1951, pp. 409 et suiv.; R. L. Koehl,“Colonialism inside Germany: 1886-1918”, The Journal of Modern History, t. 25/3, 1953, pp. 255-272 ; Henri Smotkine, «La colonisation allemande en Pologne prussienne de 1885 à 1887 », Annales ESC, t. 23/5, p. 1168-1170 ;

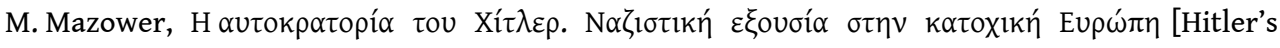
Empire : Nazi Rule in Occupied Europe], trad. grecque, Athènes 2009, pp. 19-23.

11. M. Mazower, op. cit., p. 21 ; cf. E. Hobsbawm, L’ère des Empires, trad. française, Paris 1989, p. 201.

12. AMAE, 1877/A.5, Petros Logothetis (consul général de Thessalonique) au ministère des Affaires étrangères, Thessalonique, 8 juin 1883 (réf. 709) ; pour plus de détails, voir Sp. Karavas, op. cit., pp. 117-118.

13. Rapport de A. Saktouris du 6 mars 1909, supra, n. 6.

14. Voir, à titre indicatif, D. Dakin, op. cit., pp. 534-537.

\section{RÉSUMÉS}

Comment « sauver » la Macédoine des Bulgares ? Le consul de Grèce à Serrès, Sachtouris, voit là, en 1909, un problème autant agraire que national. À cette question qui obsède les politiques grecs 
de son temps, il croit trouver une solution : acheter des tchifliks qui seraient ensuite partagés entre des paysans que les propriétaires hellènes se chargeraient "d'helléniser ». Conjoncture difficile, hausse des prix, manque de volonté des financiers, son plan reste inappliqué, en revanche les Jeunes-Turcs achèteront des terres pour « turquifier »... la Macédoine.

How to « save » Macedonia from Bulgarians? The Greek consul at Serres, Sachtouris, sees in that question, in 1909, a problem that is national as much as it is agrarian. To this particular question, that obsesses Greek politician of his time, he believes that he has found a solution: buy tchifliks which would be then shared among farmers that the Greek owners would then try to turn into Hellenes. Due to the difficult economic situation, the rising prices, the lack of willingness on behalf of the financial contributors, his plans was never implemented, quite the contrary, it was the Young Turks who bought land in order to turn Macedonia...into a Turkish territory.

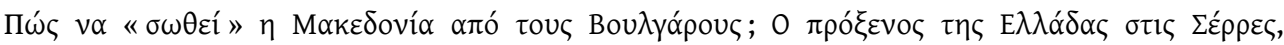

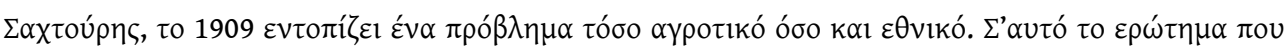

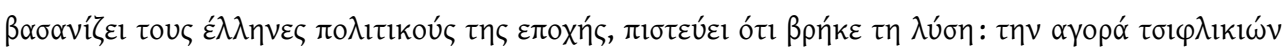

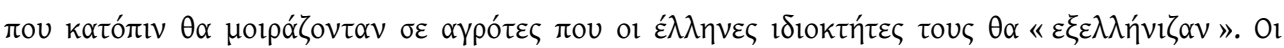

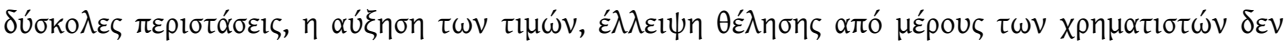

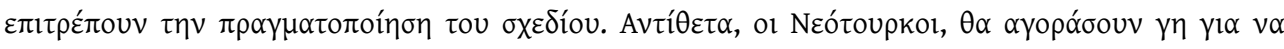

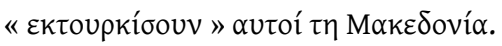

\section{INDEX}

Index géographique : Macédoine, Serrès

Thèmes : Histoire

motsclesmk МАКЕДОНИЈА, МЛАДОТУРСКАТА, ОТОМАНСКАТА ИМПЕРИЈА

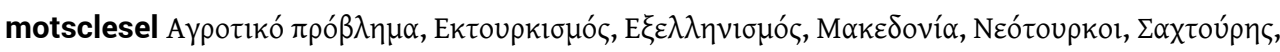

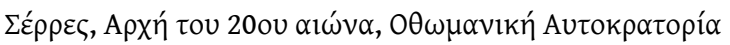

motsclestr Makedonya, Serres, Genç Türkler, Osmanlı İmparatorluğu

Mots-clés : hellénisation, Jeunes-Turcs, Nazim Bey (1870-1926), Jeunes-Turcs, Reval (rencontre) (Tallinn), réforme agraire, Sachtouris Antonios, Sandanski Yané (1872-1915), turquisation, Sevket Pacha Mahmud (1856-1913), Sandanski Yane (1872-1915), Talaat Mehmet Pacha (1874-1921), Tchiflik, IMRO/VRMO

Keywords : land reform, hellenization, Macedonia, Sachtouris Antonios, turkification, Young Turks, Sandanski Yane (1872-1915), Serres, History, twentieth century -- early, Ottoman empire

Index chronologique : vingtième siècle -- début, Empire ottoman 